\title{
On the Mixed Minus Domination in Graphs
}

\author{
Baogen Xu • Xiangyang Kong
}

Received: 6 April 2013 / Revised: 20 August 2013 / Accepted: 21 August 2013 /

Published online: 12 September 2013

(C) Operations Research Society of China, Periodicals Agency of Shanghai University, and

Springer-Verlag Berlin Heidelberg 2013

\begin{abstract}
Let $G=(V, E)$ be a graph, for an element $x \in V \cup E$, the open total neighborhood of $x$ is denoted by $N_{t}(x)=\{y \mid y$ is adjacent to $x$ or $y$ is incident with $x, y \in$ $V \cup E\}$, and $N_{t}[x]=N_{t}(x) \cup\{x\}$ is the closed one. A function $f: V(G) \cup E(G) \rightarrow$ $\{-1,0,1\}$ is said to be a mixed minus domination function (TMDF) of $G$ if $\sum_{y \in N_{t}[x]} f(y) \geqslant 1$ holds for all $x \in V(G) \cup E(G)$. The mixed minus domination number $\gamma_{t m}^{\prime}(G)$ of $G$ is defined as
\end{abstract}

$$
\gamma_{t m}^{\prime}(G)=\min \left\{\sum_{x \in V \cup E} f(x) \mid f \text { is a TMDF of } G\right\} .
$$

In this paper, we obtain some lower bounds of the mixed minus domination number of $G$ and give the exact values of $\gamma_{t m}^{\prime}(G)$ when $G$ is a cycle or a path.

Keywords Mixed minus domination function - Mixed minus domination number

\section{Introduction}

We use Bondy and Murty [1] for terminology and notation not defined here and consider finite connected simple graphs only.

Let $G$ be a graph with the vertex set $V(G)$ and edge set $E(G)$. The neighborhood of vertex $v$ is the set $\{u \mid u v \in E(G)\}$, which is denoted by $N(v)$, and $N[v]=$ $N(v) \cup\{v\}$ is the closed neighborhood of $v$. A function $f: V(G) \rightarrow\{-1,0,1\}$ is called a minus domination function (shortly MDF) of $G$, if $f[v]=f(N[v])=$

This work was supported by the National Natural Science Foundation of China (No. 11061014, 11361024, 11261019) and the Science Foundation of Jiangxi Province (No. KJLD12067).

B. Xu $(\bowtie) \cdot$ X. Kong

Department of Mathematics, East China Jiaotong University, Nanchang, Jiangxi 330013, China

e-mail: BaogenXu@163.com 
$\sum_{u \in N[v]} f(u) \geqslant 1$ for each $v \in V(G)$. It was introduced by J.E. Dunbar et al. in [2], and has been studied by other authors in [3-5]. Quite analogously, the neighborhood of edge $e$ is a set $\left\{e^{\prime} \mid e^{\prime} \in E(G)\right.$ and $e^{\prime}$ is adjacent to $\left.e\right\}$, which is denoted by $N(e)$, and $N[e]=N(e) \cup\{e\}$ is the closed edge neighborhood of $e$. We call a function $f: E(G) \rightarrow\{-1,0,1\}$ a minus edge domination function (shortly MEDF) of $G$, if $f[e]=f(N[e])=\sum_{e^{\prime} \in N[e]} f\left(e^{\prime}\right) \geqslant 1$ for each $e \in E(G)$. The weight $\omega(f)$ of $f$ is the sum of the function values of all edges in $G$. The minus edge domination number $\gamma_{m}^{\prime}(G)$ of $G$ is the minimum weight of a minus edge domination function on $G$, see [6].

In this paper, for an element $x \in V(G) \cup E(G)$, the open total neighborhood of $x$ is denoted by $N_{t}(x)=\{y \mid y$ is adjacent to $x$ or $y$ is incident with $x, y \in V(G) \cup E(G)\}$, and the closed total neighborhood of $x$ is denoted by $N_{t}[x]=N_{t}(x) \cup\{x\}$.

Definition 1.1 Let $G$ be a finite connected simple graph. A function $f: V(G) \cup$ $E(G) \rightarrow\{-1,0,1\}$ is called a mixed minus domination function (TMDF) of $G$ if $\sum_{y \in N_{t}[x]} f(y) \geqslant 1$ holds for each $x \in V(G) \cup E(G)$. The mixed minus domination number $\gamma_{t m}^{\prime}(G)$ of $G$ is defined as

$$
\gamma_{t m}^{\prime}(G)=\min \left\{\sum_{x \in V(G) \cup E(G)} f(x) \mid f \text { is a TMDF of } G\right\} .
$$

And for all totally disconnected graphs $\overline{K_{n}}$, we have $\gamma_{t m}^{\prime}\left(\overline{K_{n}}\right)=n$.

We call the mixed minus domination function of weight $\gamma_{t m}^{\prime}(G)$ a $\gamma_{t m}^{\prime}$-function of $G$.

\section{Some Lower Bounds}

Theorem 2.1 For any graph $G$ with $n$ vertices and $m$ edges,

$$
\gamma_{t m}^{\prime}(G) \geqslant\left\lceil\frac{\delta+1-\Delta}{\delta+1+\Delta}(n+m)\right\rceil
$$

where $\delta=\delta(G)$ and $\Delta=\Delta(G)$ denote the minimum and maximum degree of $G$ respectively.

Proof Let $f$ be a $\gamma_{t m}^{\prime}$-function of $G=G(V, E)$ and let

$$
\begin{aligned}
E_{1} & =\{e \in E(G) \mid f(e)=1\}, & & V_{1}=\{v \in V(G) \mid f(v)=1\}, \\
E_{0} & =\{e \in E(G) \mid f(e)=0\}, & & V_{0}=\{v \in V(G) \mid f(v)=0\}, \\
E_{-1} & =\{e \in E(G) \mid f(e)=-1\}, & & V_{-1}=\{v \in V(G) \mid f(v)=-1\} .
\end{aligned}
$$

We have

$$
\begin{aligned}
\sum_{x \in E(G) \cup V(G)} f[x]= & \sum_{e=u v \in E(G)} f[e]+\sum_{v \in V(G)} f[v] \\
= & \sum_{u v=e \in E_{1}}(d(u)+d(v)+1)-\sum_{u v=e \in E_{-1}}(d(u)+d(v)+1) \\
& +\sum_{v \in V_{1}}(2 d(v)+1)-\sum_{v \in V_{-1}}(2 d(v)+1) .
\end{aligned}
$$


For each $x \in V(G) \cup E(G)$, by Definition 1.1, $f[x]=\sum_{y \in N_{t}[x]} f(y) \geqslant 1$ and hence

$$
\begin{aligned}
|E(G)|+|V(G)| \leqslant & \sum_{x \in E(G) \cup V(G)} f[x] \leqslant\left|V_{1}\right|(2 \Delta+1)-\left|V_{-1}\right|(2 \delta+1) \\
& +\left|E_{1}\right|(2 \Delta+1)-\left|E_{-1}\right|(2 \delta+1) \\
= & \left(\left|V_{1}\right|+\left|E_{1}\right|\right)(2 \Delta+1)-\left(\left|V_{-1}\right|+\left|E_{-1}\right|\right)(2 \delta+1) .
\end{aligned}
$$

Note that $\left|V_{-1}\right|+\left|E_{-1}\right|=|V|+|E|-\left(\left|V_{1}\right|+\left|E_{1}\right|\right)-\left(\left|V_{0}\right|+\left|E_{0}\right|\right)$, then

$$
\begin{aligned}
2(\delta+1)(|E(G)|+|V(G)|) & \leqslant 2(\Delta+\delta+1)\left(\left|V_{1}\right|+\left|E_{1}\right|\right)+(2 \delta+1)\left(\left|V_{0}\right|+\left|E_{0}\right|\right) \\
& \leqslant(\Delta+\delta+1)\left[2\left(\left|V_{1}\right|+\left|E_{1}\right|\right)+\left(\left|V_{0}\right|+\left|E_{0}\right|\right)\right] .
\end{aligned}
$$

From this inequality we get

$$
2\left(\left|V_{1}\right|+\left|E_{1}\right|\right)+\left(\left|V_{0}\right|+\left|E_{0}\right|\right) \geqslant \frac{2(\delta+1)}{\Delta+\delta+1}(|V|+|E|) .
$$

Since $\gamma_{t m}^{\prime}(G)=\left(\left|V_{1}\right|+\left|E_{1}\right|\right)-\left(\left|V_{-1}\right|+\left|E_{-1}\right|\right)=2\left(\left|V_{1}\right|+\left|E_{1}\right|\right)+\left(\left|V_{0}\right|+\left|E_{0}\right|\right)-$ $(|V|+|E|)$, we have

$$
\gamma_{t m}^{\prime}(G) \geqslant \frac{2(\delta+1)}{\Delta+\delta+1}(|V|+|E|)-(|V|+|E|)=\frac{\delta-\Delta+1}{\delta+\Delta+1}(n+m) .
$$

This proof is complete.

Corollary For any $r$-regular graph $G$ with $n$ vertices, we have $\gamma_{t m}^{\prime}(G) \geqslant\left\lceil\frac{r+2}{2(2 r+1)} n\right\rceil$.

Theorem 2.2 For any graph $G$ with $n$ vertices and $m$ edges,

$$
\gamma_{t m}^{\prime}(G) \geqslant \sqrt{4(m+n)}-(m+n) .
$$

Proof Let $f$ be a $\gamma_{t m}^{\prime}$-function of $G$ and let

$$
\begin{aligned}
& E_{1}=\{e \in E(G) \mid f(e)=1\}, \quad V_{1}=\{v \in V(G) \mid f(v)=1\}, \\
& E_{0}=\{e \in E(G) \mid f(e)=0\}, \quad V_{0}=\{v \in V(G) \mid f(v)=0\}, \\
& E_{-1}=\{e \in E(G) \mid f(e)=-1\}, \quad V_{-1}=\{v \in V(G) \mid f(v)=-1\}, \\
& S_{1}=V_{1} \cup E_{1}, \quad S_{0}=V_{0} \cup E_{0}, \quad S_{-1}=V_{-1} \cup E_{-1}, \\
& \left|S_{1}\right|+\left|S_{0}\right|+\left|S_{-1}\right|=|V|+|E|=m+n .
\end{aligned}
$$

Let $\left|S_{1}\right|=p,\left|S_{0}\right|=q,\left|S_{-1}\right|=r$, then $\gamma_{t m}^{\prime}(G)=p-r=(m+n)-2 r-q$. By Definition 1.1, for each $x \in S_{-1}=\left\{x_{1}, x_{2}, \ldots, x_{r}\right\},\left|N_{t}[x] \cap S_{1}\right| \geqslant 1+\left|N_{t}[x] \cap S_{-1}\right|$, then we have

$$
r \leqslant r+\sum_{i=1}^{r}\left|N_{t}\left[x_{i}\right] \cap S_{-1}\right| \leqslant \sum_{i=1}^{r}\left|N_{t}\left[x_{i}\right] \cap S_{1}\right| .
$$

For each $y \in S_{1}=\left\{y_{1}, y_{2}, \ldots, y_{p}\right\},\left|N_{t}[y] \cap S_{1}\right| \geqslant\left|N_{t}[y] \cap S_{-1}\right|$, hence

$$
\sum_{j=1}^{p}\left|N_{t}\left[y_{j}\right] \cap S_{-1}\right| \leqslant \sum_{j=1}^{p}\left|N_{t}\left[y_{j}\right] \cap S_{1}\right| \text {. }
$$


Note that $\sum_{i=1}^{r}\left|N_{t}\left[x_{i}\right] \cap S_{1}\right|=\sum_{j=1}^{p}\left|N_{t}\left[y_{j}\right] \cap S_{-1}\right|$ and $\sum_{j=1}^{p}\left|N_{t}\left[y_{j}\right] \cap S_{1}\right| \leqslant$ $p(p-1)$, then from (2.1) and (2.2) we get $r \leqslant p(p-1)$, that is, $p \geqslant \frac{1+\sqrt{4 r+1}}{2}$. Let $\chi=\sqrt{4 r+1}$.

Note that $|E|+|V|=p+q+r \geqslant \frac{1+\sqrt{4 r+1}}{2}+r=\frac{1+\chi}{2}+\frac{\chi^{2}-1}{4}=\frac{(\chi+1)^{2}}{4}$, thus $\chi \leqslant \sqrt{4(|V|+|E|)}-1$ and $\chi \geqslant 1$, we have

$$
\begin{aligned}
\gamma_{t m}^{\prime}(G) & =p-r \geqslant \frac{1+\sqrt{4 r+1}}{2}-r \\
& =\frac{1+\chi}{2}-\frac{\chi^{2}-1}{4}=1-\frac{(\chi-1)^{2}}{4} \\
& \geqslant \sqrt{4(|V|+|E|)}-(|V|+|E|) .
\end{aligned}
$$

We have completed the proof of Theorem 2.2.

\section{Some Special Classes of Graphs}

In order to show that the bound we have got in Theorem 2.1 is sharp, we give two theorems as follows.

Theorem 3.1 For any cycle $C_{n}(n \geqslant 3)$,

$$
\gamma_{t m}^{\prime}\left(C_{n}\right)=\left\lceil\frac{2 n}{5}\right\rceil \text {. }
$$

Proof By Theorem 2.1, we have $\gamma_{t m}^{\prime}\left(C_{n}\right) \geqslant\left\lceil\frac{2 n}{5}\right\rceil$.

In order to prove that $\gamma_{t m}^{\prime}\left(C_{n}\right) \leqslant\left\lceil\frac{2 n}{5}\right\rceil$, let $V\left(C_{n}\right)=\left\{v_{1}, v_{2}, \ldots, v_{n}\right\}=\left\{v_{i} \mid 1 \leqslant i \leqslant\right.$ $n\}$ and $E\left(C_{n}\right)=\left\{e_{1}=v_{1} v_{2}, e_{2}=v_{2} v_{3}, \ldots, e_{n}=v_{n} v_{1}\right\}=\left\{e_{j} \mid 1 \leqslant j \leqslant n\right\}$.

For each $x \in V \cup E$, we know that $\left|N_{t}[x]\right|=5$. We construct a mixed minus domination function $f$ of $C_{n}$ as follows: if $n=3,4$, it is easy to prove. So we assume that $n \geqslant 5$, and define $f: V\left(C_{n}\right) \cup E\left(C_{n}\right) \rightarrow\{-1,0,1\}$ as follows:

$$
\begin{aligned}
& f\left(v_{i}\right)= \begin{cases}1 & \text { when } i \equiv 1(\bmod 5) \\
0 & \text { otherwise }\end{cases} \\
& f\left(e_{j}\right)= \begin{cases}1 & \text { when } j \equiv 3(\bmod 5) \\
0 & \text { otherwise }\end{cases}
\end{aligned}
$$

It is easy to show that the function $f$ is a mixed minus domination function of $C_{n}$ for each $n$ and $\sum_{x \in V\left(C_{n}\right) \cup E\left(C_{n}\right)} f(x)=\left\lceil\frac{2 n}{5}\right\rceil$. That is, $\gamma_{t m}^{\prime}\left(C_{n}\right) \leqslant$ $\sum_{x \in V\left(C_{n}\right) \cup E\left(C_{n}\right)} f(x)=\left\lceil\frac{2 n}{5}\right\rceil$. By far we have completed the proof of Theorem 3.1.

Theorem 3.2 Let $P_{n}$ be the path with $n \geqslant 3$ vertices, then

$$
\gamma_{t m}^{\prime}\left(P_{n}\right)= \begin{cases}\left\lceil\frac{2 n-3}{5}\right\rceil & \text { when } n \equiv 0 \text { or } 2 \text { or } 3(\bmod 5), \\ \left\lceil\frac{2 n-3}{5}\right\rceil+1 & \text { when } n \equiv 1 \text { or } 4(\bmod 5) .\end{cases}
$$


Proof It is easy to compute that this theorem holds for $n=3$ or 4 . So we assume $n \geqslant 5$.

Let $V\left(P_{n}\right)=\left\{v_{1}, v_{2}, \ldots, v_{n}\right\}=\left\{v_{i} \mid 1 \leqslant i \leqslant n\right\}, E\left(P_{n}\right)=\left\{e_{1}=v_{1} v_{2}, e_{2}=\right.$ $\left.v_{2} v_{3}, \ldots, e_{n-1}=v_{n-1} v_{n}\right\}=\left\{e_{j} \mid 1 \leqslant j \leqslant n-1\right\}$, and let $f$ be a $\gamma_{t m}^{\prime}$-function of $P_{n}$. By Definition 1.1, we have

$$
\sum_{x \in V(G) \cup E(G)} f[x]=\sum_{v \in V(G)} f[v]+\sum_{e \in E(G)} f[e] \geqslant n+n-1=2 n-1 .
$$

Since $\left|N_{t}\left[v_{1}\right]\right|=\left|N_{t}\left[v_{n}\right]\right|=3,\left|N_{t}\left[e_{1}\right]\right|=\left|N_{t}\left[e_{n-1}\right]\right|=4$, and $\left|N_{t}[x]\right|=5$ for each $x \in V(G) \cup E(G) \backslash\left\{v_{1}, v_{n}, e_{1}, e_{n-1}\right\}$, we use the same notations in the proof of Theorem 2.1 and consider the following six cases.

(1) If $f\left(v_{1}\right)+f\left(v_{n}\right)=2$, then

$$
\sum_{x \in V \cup E} f[x] \leqslant 5\left(\left|V_{1}\right|-2\right)+6-5\left|V_{-1}\right|+5\left|E_{1}\right|-5\left|E_{-1}\right|-8=5 \gamma_{t m}^{\prime}\left(P_{n}\right)-2 .
$$

(2) If $f\left(v_{1}\right)+f\left(v_{n}\right)=1$, then

$$
\begin{aligned}
\sum_{x \in V \cup E} f[x] & \leqslant 5\left(\left|V_{1}\right|-1\right)+3-5\left|V_{-1}\right|+5\left|E_{1}\right|-5\left(\left|E_{-1}\right|-1\right)-4 \\
& =5 \gamma_{t m}^{\prime}\left(P_{n}\right)-1 .
\end{aligned}
$$

(3) If $f\left(v_{1}\right)=-1, f\left(v_{n}\right)=1$ or $f\left(v_{1}\right)=1, f\left(v_{n}\right)=-1$, then

$$
\begin{aligned}
\sum_{x \in V \cup E} f[x] & \leqslant 5\left(\left|V_{1}\right|-1\right)-5\left(\left|V_{-1}\right|-1\right)+5\left(\left|E_{1}\right|-1\right)-5\left(\left|E_{-1}\right|-1\right) \\
& =5 \gamma_{t m}^{\prime}\left(P_{n}\right) .
\end{aligned}
$$

(4) If $f\left(v_{1}\right)=f\left(v_{n}\right)=0$, then

$$
\sum_{x \in V \cup E} f[x] \leqslant 5\left|V_{1}\right|-5\left|V_{-1}\right|+5\left|E_{1}\right|-5\left|E_{-1}\right|=5 \gamma_{t m}^{\prime}\left(P_{n}\right) .
$$

(5) If $f\left(v_{1}\right)=-1, f\left(v_{n}\right)=0$ or $f\left(v_{1}\right)=0, f\left(v_{n}\right)=-1$, then

$$
\begin{aligned}
\sum_{x \in V \cup E} f[x] & \leqslant 5\left|V_{1}\right|-5\left(\left|V_{-1}\right|-1\right)-3+5\left(\left|E_{1}\right|-1\right)-5\left|E_{-1}\right|+4 \\
& =5 \gamma_{t m}^{\prime}\left(P_{n}\right)+1 .
\end{aligned}
$$

(6) If $f\left(v_{1}\right)+f\left(v_{n}\right)=-2$, then

$$
\begin{aligned}
\sum_{x \in V \cup E} f[x] & \leqslant 5\left|V_{1}\right|-5\left(\left|V_{-1}\right|-2\right)-6+5\left(\left|E_{1}\right|-2\right)-5\left|E_{-1}\right|+8 \\
& =5 \gamma_{t m}^{\prime}\left(P_{n}\right)+2 .
\end{aligned}
$$

From these cases, we can see that $\sum_{x \in V \cup E} f[x] \leqslant 5 \gamma_{t m}^{\prime}\left(P_{n}\right)+2$, so we have $2 n-$ $1 \leqslant 5 \gamma_{t m}^{\prime}\left(P_{n}\right)+2$, and hence $\gamma_{t m}^{\prime}\left(P_{n}\right) \geqslant\left\lceil\frac{2 n-3}{5}\right\rceil$.

On the other hand, we will define a mixed minus domination function $f$ of $P_{n}$ as follows:

Case $1 \quad n \equiv 0,2,3(\bmod 5)$. 
Subcase 1.1 When $n \equiv 0,3(\bmod 5)$, define a function $f$ as follows:

$$
\begin{aligned}
& f\left(v_{i}\right)= \begin{cases}1 & \text { when } i \equiv 2(\bmod 5) \\
0 & \text { otherwise }\end{cases} \\
& f\left(e_{j}\right)= \begin{cases}1 & \text { when } j \equiv 4(\bmod 5) \\
0 & \text { otherwise }\end{cases}
\end{aligned}
$$

Subcase 1.2 When $n \equiv 2(\bmod 5)$, define a function $f$ as follows:

$$
\begin{aligned}
& f\left(v_{i}\right)= \begin{cases}1 & \text { when } i \equiv 2(\bmod 5), \\
0 & \text { otherwise }\end{cases} \\
& f\left(e_{j}\right)= \begin{cases}1 & \text { when } j \equiv 4(\bmod 5), \\
0 & \text { otherwise }\end{cases}
\end{aligned}
$$

It is easy to compute that $\sum_{x \in V \cup E} f[x] \leqslant\left\lceil\frac{2 n-3}{5}\right\rceil$, obviously $f$ be a mixed minus domination function of $P_{n}$ and $\gamma_{t m}^{\prime}\left(P_{n}\right) \leqslant\left\lceil\frac{2 n-3}{5}\right\rceil$. Hence we have $\gamma_{t m}^{\prime}\left(P_{n}\right)=\left\lceil\frac{2 n-3}{5}\right\rceil$ for each $n \equiv 0,2,3(\bmod 5)$.

Case $2 \quad n \equiv 1,4(\bmod 5)$.

Subcase 2.1 When $n \equiv 1(\bmod 5)$, note that for any mixed minus domination function $f$ of $P_{n}, \sum_{x \in V \cup E} f(x)=\sum_{i \equiv 1(\bmod 5)} f\left[v_{i}\right]+\sum_{j \equiv 3(\bmod 5)} f\left[e_{j}\right]+f\left[e_{n-1}\right] \geqslant$ $\frac{2 n-2}{5}+1=\left\lceil\frac{2 n-3}{5}\right\rceil+1$, so we deduce that $\gamma_{t m}^{\prime}\left(P_{n}\right) \geqslant\left\lceil\frac{2 n-3}{5}\right\rceil+1$. Define a function $f$ on $V\left(P_{n}\right) \cup E\left(P_{n}\right)$ as follows:

$$
\begin{aligned}
& f\left(v_{i}\right)= \begin{cases}1 & \text { when } i \equiv 2(\bmod 5) \\
0 & \text { otherwise }\end{cases} \\
& f\left(e_{j}\right)= \begin{cases}1 & \text { when } j \equiv 4(\bmod 5) \\
0 & \text { otherwise }\end{cases}
\end{aligned}
$$

We can see that $\sum_{x \in V \cup E} f[x] \leqslant\left\lceil\frac{2 n-3}{5}\right\rceil+1$, obviously $f$ is a mixed minus domination function of $P_{n}$ and $\gamma_{t m}^{\prime}\left(P_{n}\right) \leqslant\left\lceil\frac{2 n-3}{5}\right\rceil+1$. Hence we have $\gamma_{t m}^{\prime}\left(P_{n}\right)=$ $\left\lceil\frac{2 n-3}{5}\right\rceil+1$ for each $n \equiv 1(\bmod 5)$.

Subcase 2.2 When $n \equiv 4(\bmod 5)$, since for any mixed minus domination function $f$ of $P_{n}, \sum_{x \in V \cup E} f(x)=\sum_{i \equiv 1(\bmod 5)} f\left[v_{i}\right]+\sum_{j \equiv 3(\bmod 5)} f\left[e_{j}\right] \geqslant \frac{2 n+2}{5}=\left\lceil\frac{2 n-3}{5}\right\rceil+$ 1 , so we deduce that $\gamma_{t m}^{\prime}\left(P_{n}\right) \geqslant\left\lceil\frac{2 n-3}{5}\right\rceil+1$. We define a function $f$ on $V\left(P_{n}\right) \cup$ $E\left(P_{n}\right)$ as follows:

$$
\begin{aligned}
& f\left(v_{i}\right)= \begin{cases}1 & \text { when } i \equiv 2(\bmod 5) \\
0 & \text { otherwise }\end{cases} \\
& f\left(e_{j}\right)= \begin{cases}1 & \text { when } j \equiv 4(\bmod 5) \\
0 & \text { otherwise }\end{cases}
\end{aligned}
$$


In this case $\sum_{x \in V \cup E} f[x] \leqslant\left\lceil\frac{2 n-3}{5}\right\rceil+1$, obviously $f$ is a mixed minus domination function of $P_{n}$ and $\gamma_{t m}^{\prime}\left(P_{n}\right) \leqslant\left\lceil\frac{2 n-3}{5}\right\rceil+1$. Hence we have $\gamma_{t m}^{\prime}\left(P_{n}\right)=\left\lceil\frac{2 n-3}{5}\right\rceil+1$ for each $n \equiv 4(\bmod 5)$. The proof is complete.

Acknowledgements The authors are grateful to the referees for their careful reading with corrections and especially the referee who draws our attention to the proof in Theorem 2.2, which let us improve the proof of Theorem 2.2, and correct this lower bound.

\section{References}

[1] Bondy, J.A., Murty, V.S.R.: Graph Theory with Applications. Elsevier, Amsterdam (1976)

[2] Dunbar, J.E., Hedetniemi, S.T., Henning, M.A., McRae, A.: Minus domination in graphs. Discrete Math. 199, 35-47 (1999)

[3] Lee, J., Sohn, M.Y., Kim, H.K.: A note on graphs with large girth and small minus domination number. Discrete Appl. Math. 91(1/2/3), 299-303 (1999)

[4] Xu, B.: On minus domination and signed domination in graphs. J. Math. Res. Expo. 23(4), 586-590 (2003)

[5] Kang, L., Qiao, H., Shan, E., Du, D.: Lower bounds on the minus domination and $k$-subdomination numbers. Theory Comput. Sci. 296, 89-98 (2003)

[6] Xu, B., Zhou, S.: On minus edge domination in graphs. J. Jiangxi Norm. Univ. Natur. Sci. Ed. 31(1), 21-24 (2007). (In Chinese)

[7] Xu, B.: Two classes of edge domination in graphs. Discrete Appl. Math. 154(10), 1541-1546 (2006) 